\title{
MALLEIN AS AN AID TO THE DIAGNOSIS OF GLANDERS.
}

By J. M`Fadyean, M.B., B.Sc., Royal Veterinary College, London. IN a paper which appeared in last number of the Journal Mr Hunting and I published an account of 39 experiments or observations bearing on the value of mallein in the diagnosis of glanders. Mainly through the kind co-operation of veterinary surgeons practising in London I am now enabled to add a further series of 50 cases. In the majority of these cases the mallein used was prepared by me in the Pathological Laboratory at the Royal Veterinary College; a few of the horses were injected with mallein kindly supplied by Dr Roux of the Pasteur Institute, Paris, and the remainder with Foth's "dry mallein " procured for me by Mr John A. W. Dollar. It may here be noted that, although carefully looked for, no constant difference of effect either in the local or general reaction was observed in the three different samples of mallein.

CASE I.-Chestnut mare. Admitted into hospital on account of lameness and swelling of the off hind leg.

\begin{tabular}{|c|c|c|c|c|c|}
\hline Date. & Hour. & Pulse. & Resp. & Temp. & Remarks. \\
\hline Dec. 8 & $12.30 \mathrm{p.m}$ & 40 & 20 & $99^{\circ} 6$ & Received $21 / 2 \mathrm{cc}$. mallein. \\
\hline$"$ & 7.30 p.m. & 40 & I8 & $100^{\circ} 0$ & \\
\hline$" n$ & I I.3O p.m. & 40 & I8 & $100 \% 2$ & Swelling at seat of inoculation \\
\hline$\Rightarrow 9$ & $3.3^{\circ}$ a.m. & 42 & I6 & ror'o & $\begin{array}{l}\text { never exceeded the size of a } \\
\text { walnut. }\end{array}$ \\
\hline$"$ & 7.30 a.m. & 38 & I6 & $100^{\circ} \mathrm{O}$ & \\
\hline$"$, & I I.o a.m. & 40 & $\ldots$ & $100 \%$ & \\
\hline
\end{tabular}

I 4th March.-This mare is now at work, and apparently in good health.

CASE II.-Grey mare. Admitted into hospital on account of a suspicious red speck visible on the off nostril and inflammatory thickening of the near hind leg.

\begin{tabular}{|c|c|c|c|c|c|}
\hline Date. & Hour. & Putse & Resp. & Temp.: & Remarks. \\
\hline Dec. 8 & I 2.0 noon & 44 & I 8 & $99 \cdot 8$ & \multirow{6}{*}{$\begin{array}{l}\text { Received } 21 / 2 \text { cc. mallein. } \\
\text { Animal continued to feed well. } \\
\text { Local swelling reached the } \\
\text { size of an orange, and was } \\
\text { tender on pressure. }\end{array}$} \\
\hline & 6.20 p.m. & 48 & 20 & $99^{\circ} 8$ & \\
\hline$"$ & 10.20 p.m. & 42 & 14 & $100 \cdot 6$ & \\
\hline & 2.20 a.m. & 40 & I 5 & $100^{\circ}$ & \\
\hline & $6.20 \mathrm{a} . \mathrm{m}$ & 36 & I 5 & $100^{\circ}$ & \\
\hline & II.o a.m. & 40 & $\ldots$ & $100^{\circ} 0$ & \\
\hline
\end{tabular}

I 4 th March.-Mare at work, and apparently healthy. 
CASE III.-Grey mare. Admitted into hospital on 23rd November on account of fistulous withers. At that time looked well and showed no symptoms of either farcy or glanders. A few days prior to the date of injection with mallein a suspicious "bud" formed on the inside of the off knee.

\begin{tabular}{|c|c|c|c|c|c|}
\hline Date. & Hour. & Pulse. & Resp. & Temp. & Remarks. \\
\hline Dec. 8 & 12.30 p.m. & 68 & I8 & $103^{\circ} 0$ & $\begin{array}{l}\text { Received a dose of German } \\
\text { mallein. }\end{array}$ \\
\hline$" \#$ & 7.20 p.m. & 74 & 18 & $103^{\circ} 5$ & $\begin{array}{l}\text { Local swelling as large as an } \\
\text { orange. }\end{array}$ \\
\hline$" \#$ & I I. 20 p.m. & 76 & 16 & $104: 2$ & $\begin{array}{l}\text { Local swelling measures } 6 \text { inches } \\
\text { across. }\end{array}$ \\
\hline$" 9$ & 3.20 a.m. & 82 & 16 & 103.5 & Local swelling has spread as far \\
\hline & 7.20 a.m. & 76 & I6 & $104 \%$ & $\begin{array}{l}\text { as the jugular furrow; ant- } \\
\text { mal depressed, and not } \\
\text { feeding. }\end{array}$ \\
\hline$"$ & II.O a.m. & 75 & ... & 103.8 & \\
\hline "IO & IO.o a.m. & 70 & $\ldots$ & $102 \cdot 6$ & $\begin{array}{l}\text { Swelling gone. Not feeding } \\
\text { well. }\end{array}$ \\
\hline
\end{tabular}

Slaughtered I 2 th January, and found to be badly glandered.

CASE IV.-Roan mare; isolated on 2Ist November I892, on account of a swollen arm, enlarged submaxillary gland, and a nasal discharge. At the date of injection there were some farcy buds on the front of the off arm. General condition good.

\begin{tabular}{|c|c|c|c|c|c|}
\hline Date. & Hour. & Pulse. & Resp. & Temp. & Remarks. \\
\hline Dec. 18 & 5.3o p.m. & 60 & 12 & $102^{\circ} \mathrm{O}$ & Received $21 / 2$ cc. mallein. \\
\hline$"$ & 9.30 p.m. & 56 & IO & IOI 8 & Animal continued to feed well, \\
\hline$"$ Ig & I.30 a.m. & 58 & 14 & $102 \cdot 6$ & $\begin{array}{l}\text { but local reaction was very } \\
\text { great, the swelling measur- }\end{array}$ \\
\hline$"$ & 5.30 a.m. & 62 & 20 & $103^{\circ} \mathrm{O}$ & $\begin{array}{l}\text { ing } 9 \text { inches across and } \\
\text { reaching as far as the jugu- }\end{array}$ \\
\hline " & $9.30 \mathrm{a} . \mathrm{m}$. & 56 & 13 & $102^{\circ} \circ$ & \\
\hline
\end{tabular}

The animal was slaughtered on the $29^{\text {th }}$ December and found to be bädly glandered.

CASE V.-Brown horse. Admitted into hospital on account of an abscess in the intermaxillary space. At date of injection petechial 
spots were present on nostril, conjunctiva, and labial mucous membrane.

\begin{tabular}{|c|c|c|c|c|c|c|}
\hline Date. & & Hour. & Pulse. & Resp. & Temp. & Remarks. \\
\hline Dec. & & I 2.30 p.m. & $5^{6}$ & Io & $101 \cdot 8$ & Received $21 / 2$ cc. mallein. \\
\hline & " & 6.40 p.m. & 72 & I 2 & I0 $3 \cdot 8$ & \\
\hline " & " & I0.40 p.m. & 74 & I4 & 1048 & \\
\hline$"$ & 9 & 2.40 a.m. & 68 & 12 & 103.8 & $\begin{array}{l}\text { Swelling at seat of inoculation } \\
\text { considerable, measuring } 6\end{array}$ \\
\hline " & $"$ & 6.40 a.m. & 62 & 12 & $102 * 5$ & $\begin{array}{l}\text { inches across ; animal a } \\
\text { little off feed. }\end{array}$ \\
\hline$"$ & $"$ & II.o a.m. & 70 & $\cdots$ & 103.8 & \\
\hline, 1 & 10 & II.O a.m. & 68 & $\cdots$ & $102 \%$ & \\
\hline
\end{tabular}

I 6 th Feb.-The horse was slaughtered on this date and found to be glandered.

CASE VI.-A grey mare ; had been trephined by $\mathrm{Mr}$ Rogerson on the 6th August 1892 for an obstinate, bilateral, foetid, nasal discharge. She also had slight tumefaction of the submaxillary glands. For a short time after the operation the case did well, but when the syringing of the sinuses was stopped and the wounds were allowed to heal the discharge returned. For the following three months the mare was isolated, and during that time localised swellings about the limbs and under the abdomen appeared on several occasions. After exercise the respiration became distressed and was attended by a snuffling noise in both nostrils.

\begin{tabular}{|c|c|c|c|c|c|c|}
\hline \multicolumn{2}{|c|}{ Date. } & Hour. & Pulse. & $R \operatorname{csp}$. & Temp. & Remarks. \\
\hline Dec. & I 8 & 6.0 p.m. & 54 & $\mathrm{~J}_{4}$ & IOI'O & Received $2 \mathrm{x} / 2$ cc. mallein. \\
\hline$"$ & $"$ & IO.O p.m. & 50 & 20 & $101 \cdot 5$ & \\
\hline$"$ & & 2.0 a.m. & 54 & 18 & $102^{\circ} \mathrm{I}$ & $\begin{array}{l}\text { Mare continued to feed well; } \\
\text { local swelling at maximum }\end{array}$ \\
\hline , & " & 6.0 a.m. & 52 & 20 & 1015 & measured $6 \times 4$ inches. \\
\hline$"$ & $"$ & Io.O a.m. & 46 & 20 & $100 \cdot 8$ & \\
\hline
\end{tabular}

The mare was slaughtered about a fortnight later. The post-mortem revealed extensive suppurative disease of the sinuses and turbinated bones, but no trace of glanders. 
CASE VII.-Grey horse ; admitted to hospital on account of injured foot. An abscess subsequently developed under the jaw.

\begin{tabular}{|c|c|c|c|c|c|c|}
\hline Date & & Hour. & Pulse. & $\operatorname{Resp}$ & Temp. & Remarks. \\
\hline Mar. & & I2.0 noon & 48 & 12 & $99^{\circ} 8$ & Received $21 / 2$ cc. mallein. \\
\hline " & $"$ & 6.0 p.m. & 56 & I 2 & 100.6 & \\
\hline$"$ & $"$ & IO.o p.m. & $5 \mathrm{I}$ & 12 & $100 \% 2$ & $\begin{array}{l}\text { Animal continued to feed well, } \\
\text { and gave no evidence of con- }\end{array}$ \\
\hline & 6 & 2.0 a.m. & 50 & 12 & $100^{\circ} 0$ & $\begin{array}{l}\text { stitutional disturbance. Local } \\
\text { swelling small-about } 4 \text { inches }\end{array}$ \\
\hline - " & " & 6.0 a.m. & 44 & 12 & $99^{\circ} 6$ & across. \\
\hline$"$ & $"$ & IO.o a.m. & 42 & 12 & $99^{\circ} \mathrm{O}$ & \\
\hline
\end{tabular}

The abscess under the jaw burst and then healed. The horse subsequently did well.

CASE VIII.-Bay horse; admitted into hospital on Ist February. Had been under observation for the previous six months, owing to tumefaction of the near submaxillary gland, and unthriftiness. Slight nasal discharge.

\begin{tabular}{|c|c|c|c|c|c|c|}
\hline Date. & & Houer. & Pulse. & Resp. & Temp. & Remarks. \\
\hline Mar. 5 & & I 2.0 noon & 52 & 14 & $101^{\prime} 2$ & Received $21 / 2 \mathrm{cc}$. mallein. \\
\hline & & 6.0 p.m. & 62 & Io & $103 \%$ & \\
\hline$"$ & " & IO.O p.m. & 68 & 12 & $104 \cdot 3$ & \\
\hline$"$ & 6 & 2.0 a.m. & 64 & IO & 104.5 & Constitutional disturbance ; appe- \\
\hline$"$ & $"$ & 6.0 a.m. & 48 & 10 & 104.4 & $\begin{array}{l}\text { tite suppressed ; local swell- } \\
\text { ing extensive, } 7 \text { inches across }\end{array}$ \\
\hline$"$ & 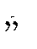 & IO.o a.m. & $\ldots$ & $\ldots$ & $\mathrm{IO}+2$ & \\
\hline$"$ & 7 & IO.O a.m. & 50 & 10 & $103^{*} 2$ & \\
\hline$"$ & 8 & IO.O a.m. & $\ldots$ & $\ldots$ & 10.46 & \\
\hline
\end{tabular}

In order to test the effect of unfiltered mallein (containing dead glanders bacilli) this horse was submitted to a second injection as follows :- 


\begin{tabular}{|c|c|c|c|c|c|}
\hline Date. & Hour. & Pulse. & Resp. & Temp. & Remarks. \\
\hline Mar. I3 & I 2.0 noon & 46 & 12 & IOI'6 & $\begin{array}{l}\text { Received } 21 / 2 \\
\text { mallein. }\end{array}$ \\
\hline & 6.0 p.m. & $5^{\circ}$ & I 2 & 103.0 & Animal off feed and thirsty. \\
\hline & IO.O p.m. & 68 & 12 & $104^{\circ} 6$ & $\begin{array}{l}\text { Local swelling extended to } \\
\text { jugular furrow and pre- }\end{array}$ \\
\hline$\eta \quad 14$ & 2.0 a.m. & 72 & I 2 & $104^{\circ} 6$ & $\begin{array}{l}\text { scapular glands, and mea- } \\
\text { sured } 7 \text { inches across. }\end{array}$ \\
\hline$" \quad "$ & 6.0 a.m. & 68 & I 2 & $104 \cdot 6$ & \\
\hline
\end{tabular}

This horse was killed on the I6th March, and found to be badly glandered.

CASE IX.-Chestnut horse; admitted into hospital on 9th January, on account of a discharge from the near nostril, swelling of the submaxillary gland, and slight thickening of the near hind leg.

\begin{tabular}{|c|c|c|c|c|c|}
\hline Date. & Hour. & Pulse. & Resp. & Temp. & Remarks. \\
\hline Mar. 5 & I 2.0 noon & 48 & I 5 & $100^{\circ} 0$ & Received $21 / 2$ cc. mallein. \\
\hline$"$ & 6.0 p.m. & 48 & I6 & $101 \cdot 2$ & \\
\hline$" \quad "$ & Io.o p.m. & 48 & 15 & $100{ }^{\circ}$ & $\begin{array}{l}\text { No constitutional disturbance ; } \\
\text { feeds well ; local swelling }\end{array}$ \\
\hline " 6 & $2.0 \mathrm{a} . \mathrm{m}$. & 48 & I3 & $100 \% 4$ & slight- $-2 \frac{1}{2}$ inches across. \\
\hline$"$ & 6.0 a.m. & 42 & 13 & $99^{\circ} 6$ & \\
\hline
\end{tabular}

I th March. - On this date Mr Rogerson trephined the frontal sinus, which he found to be filled with pus.

CASE X.-Aged mare; suspected on account of a fœetid glairy discharge from left nostril, and slight tumefaction of submaxillary glands; legs thick, coat good, does not grunt.

\begin{tabular}{|c|c|c|c|c|c|c|}
\hline \multicolumn{2}{|c|}{ Date. } & Hour. & Pulse. & Resp. & Temp. & Remarks. \\
\hline & 30 & Il.o p.m. & 40 & I 2 & $100 \cdot 0$ & Received $21 / 2 \mathrm{cc}$. mallein. \\
\hline$"$ & $3 I$ & 4.0 a.m. & 44 & I6 & $100^{\circ} 0$ & lo 1 \\
\hline " & $"$ & 6.0 a.m. & 40 & 12 & $100 \cdot 6$ & $\begin{array}{l}\text { about } 3 \text { inches in diameter, } \\
\text { and painful. }\end{array}$ \\
\hline$"$ & $"$ & 9.0 a.m. & 45 & 8 & $103^{\circ} 0$ & \\
\hline$"$ & $"$ & I2.0 noon & 48 & 9 & $104^{\circ} \mathrm{O}$ & \\
\hline " & $"$ & $4.3^{\circ}$ p.m. & 48 & 9 & $103 \cdot 8$ & $\begin{array}{l}\text { Swelling scarcely perceptible; } \\
\text { feeding well. }\end{array}$ \\
\hline$"$ & $"$ & $9.30 \mathrm{p} . \mathrm{m}$ & 44 & 9 & $102 \cdot 8$ & \\
\hline
\end{tabular}


This mare was killed on 3rd January. The septum nasi was covered with ecchymosed spots and patches, but no ulcers were present. No tubercles were discovered in the lungs, but there were numerous old pleuritic lesions.

CASE XI.-Grey cart gelding, with a large sarcomatous tumour of the left orbit. Left submaxillary gland enlarged, but not adherent. Foetid flaky discharge from left nostril.

\section{First InOCULATION.}

\begin{tabular}{|c|c|c|c|c|c|}
\hline Date. & Hour. & Resp. & Pulse. & Temp & Remarks. \\
\hline Dec. 30 & I I.O p.m. & I6 & 40 & 100.8 & Injected $2 \mathrm{I} / 2 \mathrm{cc}$. mallein. \\
\hline$\Rightarrow \quad 3 I$ & 4.0 a.m. & I6 & 40 & $100^{\circ} 4$ & $\begin{array}{l}\text { Swelling very slight at seat of } \\
\text { inoculation, but sensitive. }\end{array}$ \\
\hline$"$ & 6.0 a.m. & 12 & 44 & IOI'O & As at 4.0 a.m. \\
\hline$"$ & 9.0 a.m. & 8 & 42 & IOI'O & Had eaten feed. \\
\hline$"$ & I2.30 p.m. & $\mathrm{I}_{4}$ & 42 & IOI'O & Feeding well. \\
\hline$"$ & 4.3० p.m. & 9 & 44 & $101 \%$ & $\begin{array}{l}\text { Feeding well; swelling very sensi- } \\
\text { tive, but small. }\end{array}$ \\
\hline " & 9.30 p.m. & IO & 48 & IOI 4 & $\cdots$ \\
\hline
\end{tabular}

\section{SECOND INOCULATION.}

\begin{tabular}{|c|c|c|c|c|c|}
\hline Date. & Hour. & Resp. & Pulse. & Temp. & Remarks. \\
\hline Jan. 5 & 9.30 p.m. & 8 & 40 & $102 * 2$ & Inoculated with $2 \frac{1}{2} \mathrm{cc}$. mallein. \\
\hline, 6 & 3.0 a.m. & 8 & 40 & $100 \cdot 6$ & Swelling at seat of inoculation \\
\hline$"$ & 6.0 a.m. & 8 & 40 & IOIO & $\begin{array}{l}\text { about size of a frorin and } \\
\text { not painful ; animal feeding }\end{array}$ \\
\hline$"$ & 9.0 a.m. & 8 & 44 & IOI'O & \\
\hline
\end{tabular}

This animal was afterwards chloroformed and operated on by $\mathrm{Mr}$ Hobday, who endeavoured to excise the tumour, but the effort was fruitless on account of the large extent over which the new growth had spread, and the animal was destroyed.

Post-mortem.-Revealed no glanders lesions. 
CASE XII.-Grey hunter gelding; 7 years old; a bad roarer, but otherwise apparently healthy, except for a peculiar "dropped" condition of the spine.

FIRST INOCULATION.

\begin{tabular}{|c|c|c|c|c|c|c|}
\hline Dat & & Hour. & Resp. & Pulse. & Temp. & Remarks. \\
\hline Dec. & & I I.O p.m. & 16 & 40 & $100^{\circ} 6$ & Received $21 / 2 \mathrm{cc}$. mallein. \\
\hline & $3 \mathbf{I}$ & 4.0 p.m. & 16 & 44 & $100^{\circ} 0$ & $\begin{array}{l}\text { Swelling circular, } 2 \text { inches in } \\
\text { diameter, very sensitive. }\end{array}$ \\
\hline$"$ & $"$ & 6.0 a.m. & 12 & 36 & $100 \cdot 6$ & $\cdots$ \\
\hline$"$ & $"$ & 9.0 a.m. & 8 & 40 & $102 \cdot 8$ & $\begin{array}{l}\text { Swelling smaller but very sore. } \\
\text { Feeding well. }\end{array}$ \\
\hline$"$ & $"$ & $\mathrm{I} 2.30 \mathrm{p.m}$ & 12 & 48 & $105^{\circ} \mathrm{O}$ & $\cdots$ \\
\hline$"$ & $"$ & 4.30 p.m. & 8 & 40 & $103 \cdot 2$ & Feeding well. \\
\hline$"$ & $"$ & 9.3० p.m. & $1 ?$ & $3^{6}$ & IOI'O & No perceptible swelling. \\
\hline
\end{tabular}

SECOND InOCULATion.

\begin{tabular}{|c|c|c|c|c|c|c|}
\hline Date. & & Hour. & Resp. & Pulse. & Temp. & Remarks. \\
\hline Jan. & & 9.3० p.m. & 8 & 44 & $100^{\circ} 2$ & Received $21 / 2 \mathrm{cc}$. mallein. \\
\hline & 6 & 3.0 a.m. & 8 & 44 & IOI $\%$ & $\begin{array}{l}\text { Swelling at seat of inoculation } \\
\text { very tender ; } 3 \text { inches } \times 2 \text {. }\end{array}$ \\
\hline$"$ & $"$ & 6.0 a.m. & 8 & 44 & $100 \cdot 8$ & $\cdots$ \\
\hline$"$ & $"$ & 9.0 a.m. & IO & 44 & $102 \cdot 6$ & Swelling less, but very sensitive. \\
\hline$"$ & $"$ & 2.0 p.m. & I 2 & 48 & $102 \cdot 8$ & $\ldots$ \\
\hline & $"$ & 5.0 p.m. & 8 & 40 & $102^{\circ} \mathrm{O}$ & Swelling still tender. \\
\hline
\end{tabular}

This animal was slaughtered a few days later, but on post-mortem no glanders lesions were discoverable. 
CASE XIII.-Bay pony mare, aged; apparently healthy.

\begin{tabular}{|c|c|c|c|c|c|c|}
\hline \multicolumn{2}{|c|}{ Date. } & Hour. & Resp. & Pulse. & Temp. & Remarks. \\
\hline Jan. & & IO.O p.m. & 52 & 36 & $100^{\circ} 4$ & Received $21 / 2 \mathrm{cc}$. mallein. \\
\hline$" '$ & 13 & 4.30 a.m. & 52 & 32 & $99^{\circ} 9$ & $\begin{array}{l}\text { No swelling perceptible at seat of } \\
\text { inoculation, although the skin } \\
\text { felt slightly thickened; not at } \\
\text { all sore. Feeding well. }\end{array}$ \\
\hline$"$ & $"$ & 9.0 a.m. & 16 & 36 & $99^{\circ} 0$ & $\begin{array}{l}\text { Feeding well; no swelling; very } \\
\text { slightly sore. }\end{array}$ \\
\hline 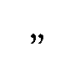 & $"$ & I 2.0 noon & 20 & 36 & $99^{\circ} \mathbf{I}$ & Feeding well ; no swelling. \\
\hline$"$ & $"$ & 5.0 p.m. & 16 & 40 & $99^{\circ} 6$ & As at i 2.0 noon. \\
\hline$"$ & $\eta$ & IO.O p.m. & 20 & 42 & $99^{\circ} 8$ & As at 12.0 noon. \\
\hline
\end{tabular}

This mare is still alive and apparently in perfect health.

CASE XIV.-Bay mare, in good condition; small indurated submaxillary gland on near side.

\begin{tabular}{|c|c|c|c|}
\hline Date. & Houer. & Temp. & Remarks. \\
\hline Mar. 2 & $9.40 \mathrm{a} . \mathrm{m}$. & 100.8 & Received $2 \frac{1}{2} \mathrm{cc}$. mallein. \\
\hline$"$ & 3.40 p.m. & $100^{\circ} 2$ & \\
\hline$"$ & 7.40 p.m. & $100: 2$ & $\begin{array}{l}\text { Little local swelling; no constitutional dis- } \\
\text { turbance. }\end{array}$ \\
\hline$" \quad$, & I I. 40 p.m. & $100 \cdot 8$ & \\
\hline
\end{tabular}

This mare is still alive and apparently well.

CASE XV.-Grey mare, in good condition; suspected on account of tumefied submaxillary gland on near side.

\begin{tabular}{|c|c|c|c|}
\hline Date. & Hour. & Temp. & Renarks. \\
\hline Mar. 2 & 9.40 a.m. & 100.8 & Received $21 / 2$ cc. mallein. \\
\hline , & 3.40 p.m. & I00: 8 & $\ldots$ \\
\hline$" \quad "$ & $7.40 \mathrm{p} . \mathrm{m}$. & $105 \%$ & $\begin{array}{l}\text { Slight rigors; much swollen at seat of } \\
\text { inoculation. }\end{array}$ \\
\hline & & & \\
\hline
\end{tabular}

This animal was slaughtered a few days later, and found to be glandered. 
CASE XVI.-Bay mare, aged; suspected on account of slight discharge from near nostril and tumefaction of the submaxillary gland on the same side.

\begin{tabular}{|c|c|c|c|}
\hline Date. & Hour. & Temp. & Remarks. \\
\hline Mar. 2 & 9.40 a.m. & $100 \cdot 8$ & Received $21 / 2 \mathrm{cc}$. mallein. \\
\hline$"$ & 3.40 p.m. & $100 \cdot 8$ & \\
\hline$"$ & 7.40 p.m. & $105^{\circ} \mathrm{O}$ & $\begin{array}{l}\text { Not feeding; shaking; seat of inoculation } \\
\text { swollen and painful. }\end{array}$ \\
\hline$"$ & I 1.40 p.m. & $105^{\circ} \mathrm{O}$ & \\
\hline
\end{tabular}

Slaughtered a few days later and found to be glandered.

CASE XVII.-Bay mare, 5 years old ; nasal discharge and tumefied gland on near side.

\begin{tabular}{|c|r|r|l|}
\hline Date. & Hour. & Temp. & \multicolumn{1}{|c|}{ Remarks. } \\
\cline { 2 - 3 } Mar. 3 & 3.0 p.m. & 101'6 & Received 21/2 cc. mallein. \\
$n+$ & I0.0 a.m. & $104 \% 0$ & Shaking; much swollen at seat of inoculation. \\
\hline
\end{tabular}

Slaughtered a few days later and found to be glandered.

CASE XVIII.-Black aged gelding. Enlarged submaxillary gland on off side; no discharge.

\begin{tabular}{|c|c|c|c|}
\hline Date. & Hour. & Temp. & Remarks. \\
\hline Mar. 3 & 3.0 p.m. & $102{ }^{\circ} 0$ & Received $21 / 2 \mathrm{cc}$. mallein. \\
\hline$" \quad "$ & IO.O a.m. & $104^{\circ} \mathrm{O}$ & Painful swelling at seat of inoculation. \\
\hline
\end{tabular}

Killed a few days later and found to be glandered.

CASE XIX.-Grey gelding, aged ; suspected on account of enlarged gland and slight nasal discharge on near side.

\begin{tabular}{|r|r|r|l|}
\hline Date. & Hour. & Temp. & \multicolumn{1}{|c|}{ Remarks. } \\
\hline Mar. 3 & 3.0 p.m. & $100^{\circ} 6$ & Injected with 2 1/2 cc. mallein. \\
\hline, & I0.0 a.m. & $105^{\circ} 0$ & Shaking; seat of inoculation much swollen. \\
\hline
\end{tabular}

Killed a few days later and found to be glandered. 
CASE XX.-Dun mare, with swelling under abdomen and between hind legs.

\begin{tabular}{|c|c|c|c|}
\hline Date. & Hour. & Temp & Remarks. \\
\hline Mar. 8 & 3.0 p.m. & $100 \cdot 6$ & Injected with $2 \frac{1}{2} \mathrm{cc}$. mallein. \\
\hline$", \quad "$ & 6.0 p.m. & IoI 6 & No local reaction \\
\hline$" \quad "$ & 9.0 p.m. & $105^{\circ}$ & \\
\hline
\end{tabular}

Killed and found to be glandered.

CASE XXI.-Brown gelding with indurated submaxillary gland.

\begin{tabular}{|c|c|c|c|}
\hline Date. & Hour. & Temp. & Remarks. \\
\hline Mar. 8 & 3.0 p.m. & 100.6 & Received $2 \mathrm{r} / 2 \mathrm{cc}$. mallein. \\
\hline ", , & 6.0 p.m. & $102 \cdot 6$ & Shalino . seat of inoculation much curollen \\
\hline$"$, & 9.0 p.m. & $105^{\circ} 0$ & \\
\hline
\end{tabular}

Killed and found to be glandered.

CASE XXII.-This was a bay mare the subject of farcy. She received the usual dose of mallein and the temperature subsequently rose from $10 I^{\circ}$ to $105 .^{\circ}$

CASE XXIII.-Bay mare, suspected on account of swollen legs.

\begin{tabular}{|c|c|c|c|}
\hline Date. & Hour. & Temp. & Remarks. \\
\hline Mar. 9 & 9.45 a.m. & IOI'O & Received $2 / 2 / 2$ cc. mallein. \\
\hline$"$ & 3.45 p.m. & $103 \cdot 6$ & \\
\hline$" \quad "$ & 9.0 p.m. & $105^{\circ} 0$ & Much swollen at seat of inoculation \\
\hline IO & 6.30 a.m. & $104 \%$ & \\
\hline$"$ & 7.0 p.m. & $103{ }^{\circ}$ & \\
\hline
\end{tabular}

Killed and found to be glandered. 
CASE XXIV.-Brown horse, in good condition.

\begin{tabular}{|c|c|c|c|}
\hline Date. & Hour. & Temp. & \multicolumn{1}{|c|}{ Remarks. } \\
\hline Mar. IO & 3.0 p.m. & Ior॰o & Received 21/2 cc. mallein. \\
", I I & 9.0 a.m. & $105 \circ$ & Shaking; much local swelling. \\
\hline
\end{tabular}

Killed and found to be glandered.

CASE XXV.—Roan gelding, aged, weak; discharging at both nostrils, and near submaxillary gland enlarged.

\begin{tabular}{|c|c|c|c|c|}
\hline Date & & Hour. & Temp. & Remarks. \\
\hline Mar. & & I 2.0 noon & $102^{\circ} 0$ & Received $21 / 2 \mathrm{cc}$. mallein. \\
\hline & " & 8.o p.m. & $104 \cdot 8$ & \\
\hline$"$ & $"$ & I2.0 midnight & $102^{\circ} \mathrm{O}$ & No swelline at seat of iniection \\
\hline " & 4 & $6.0 \mathrm{a} \mathrm{m}$ & $103^{6} 6$ & \\
\hline , & " & 12.0 noon & $102^{\circ} \mathrm{O}$ & \\
\hline
\end{tabular}

Died on the 5th March. Post-mortem revealed pulmonary abscess and oedema, but no glanders lesions.

CASE XXVI.-Black gelding, with a suspicious discharge from off nostril.

\begin{tabular}{|c|c|c|c|}
\hline Date. & Hour. & Temp. & Remarks. \\
\hline Mar. I4 & 10.30 a.m. & ror'o & Received $21 / 2 \mathrm{cc}$. mallein. \\
\hline$"$ & 4.30 p.m. & $100 \cdot 6$ & \\
\hline$"$ & 8.30 p.m. & $105^{\circ} 0$ & Seat of inoculation much swollen. \\
\hline$\Rightarrow \quad$ I 5 & 9.0 a.m. & IO4. & \\
\hline
\end{tabular}

Killed on the i6th March and found to be glandered.

CASE XXVII.-Bay mare in good condition. Very small indurated submaxillary gland on near side, but no nasal discharge.

\begin{tabular}{|c|c|c|c|}
\hline Date. & Hour. & Temp. & Remarks. \\
\hline Mar. I 4 & 4.0 p.m. & IOI'6 & Injected with $2 / 2 / 2 c$. mallein. \\
\hline$" \quad "$ & IO.o p.m. & 103.0 & \\
\hline I 5 & $2.0 \mathrm{a} . \mathrm{m}$. & $105^{\circ} \mathrm{O}$ & Large painful swelling at seat of inoculation. \\
\hline$"$, & 6.0 a.m. & $105^{\circ} \mathrm{O}$ & \\
\hline
\end{tabular}

16th March.-Killed and found to be glandered. 
CASE XXVIII.-Chestnut mare from the country, with no suspicion of glanders.

\begin{tabular}{|c|c|c|c|c|}
\hline Date. & Hour. & Pulse. & Temp. & Remarks. \\
\hline Feb. 12 & 6.0 p.m. & 32 & $100 \% 6$ & Received $21 / 2 \mathrm{cc}$. mallein. \\
\hline$"$, & I 2.0 midnight & 32 & $100 \cdot 6$ & \\
\hline$"$ I 3 & $3.0 \mathrm{a} . \mathrm{m}$. & 34 & $100 \cdot 8$ & $\begin{array}{l}\text { No general disturbance; little local } \\
\text { reaction. }\end{array}$ \\
\hline$"$, & 6.0 a.m. & 34 & $100 \cdot 8$ & \\
\hline
\end{tabular}

This mare received a second injection on the $23 \mathrm{rd}$ of February, and in that case also there was no appreciable reaction, either general or local. The mare is still alive and free from any symptoms of glanders or farcy.

CASE XXIX. - Yearling filly; affected with tympanites of the guttural pouch ; not suspected of glanders.

\begin{tabular}{|c|c|c|c|c|c|}
\hline Date. & Hour. & Resp. & Pulse. & Temp. & Remarks. \\
\hline Jan. 30 & 9.0 p.m. & I 4 & 66 & $101{ }^{2}$ & Received $21 / 2 \mathrm{cc}$. mallein. \\
\hline$" 3 I$ & $3.0 \mathrm{a} . \mathrm{m}$. & I I & $6 \mathrm{I}$ & IOI 2 & \\
\hline$"$, & 6.0 a.m. & I 5 & 60 & $101^{\circ} 2$ & $\begin{array}{l}\text { ing at seat of inoculation } \\
21 / 2 \text { inches } \times 1 \text { inch. }\end{array}$ \\
\hline$"$ & 9.0 a.m. & I 4 & 60 & $\mathrm{IOI}^{2} 2$ & \\
\hline
\end{tabular}

The filly was subsequently killed, and found to be free from glanders.

CASE XXX, - Bay gelding, with bony enlargements in various parts of body, but no suspicion of glanders.

\begin{tabular}{|c|c|c|c|c|c|}
\hline Date. & Hour. & Resp. & Pulse. & Temp. & Remarks. \\
\hline Jan. 30 & 9.0 p.m. & 18 & 38 & $102 \%$ & Received $21 / 2$ cc. mallein. \\
\hline$" 3 \mathrm{I}$ & 3.0 a.m. & 14 & 43 & $102 \%$ & \\
\hline$" \quad "$ & 6.0 a.m. & 14 & 48 & IOI 8 & $\begin{array}{l}\text { No general disturbance; local } \\
\text { swelling } 31 / 2 \times 2 \text { inches. }\end{array}$ \\
\hline ", & $9.0 \mathrm{a} . \mathrm{m}$. & 14 & 44 & Ior 4 & \\
\hline
\end{tabular}

This horse was killed on the Ist February, and the post-mortem did not reveal any lesions indicative of glanders. 
CASE XXXI.-Cab mare in fair condition; intermittent discharge from near nostril, and submaxillary gland enlarged ; suspected ozæna treated for several weeks without improvement. Injected with mallein on the I6th March.

\begin{tabular}{|c|c|}
\hline Hour. & Temperature. \\
\hline I.o p.m. & $102^{\circ} 0$ \\
\hline 7.0 p.m. & $104 \%$ \\
\hline I I.o p.m. & $104 \cdot 4$ \\
\hline 3.0 a.m. & 1042 \\
\hline 7.0 a.m. & $105^{\circ} 2$ \\
\hline I.O p.m. & 104.2 \\
\hline
\end{tabular}

Killed and found to be glandered in lungs.

CASE XXXII. - Bay mare; with no symptom of glanders, but been working with a horse that developed that disease.

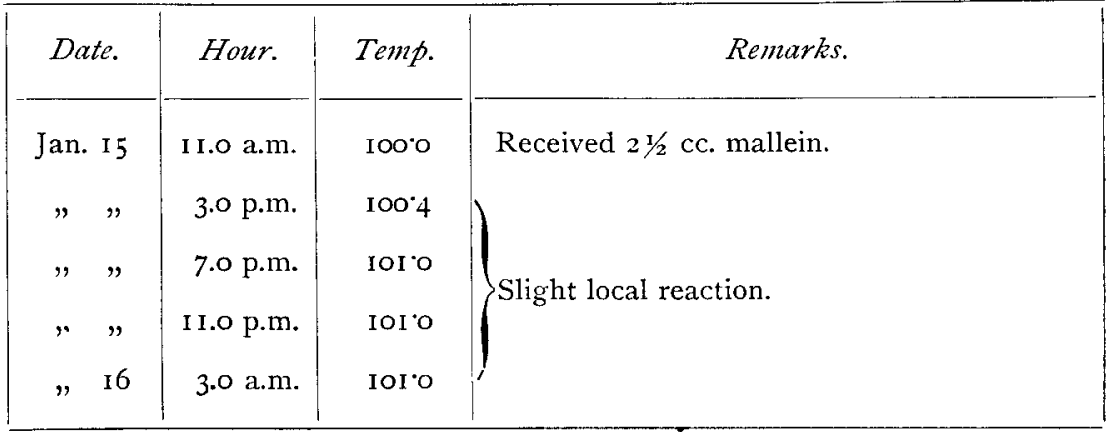

This mare met with an accident which necessitated her destruction on the 2oth of February. On post-mortem all the internal organs appeared healthy.

CASE XXXIII.-Dun horse; chronic cough and discharge from right nostril.

\begin{tabular}{|c|c|c|c|}
\hline Date. & Hour. & Temp. & Remarks. \\
\hline Jan. I 5 & I I.O a.m. & $101^{\circ} 2$ & Received $21 / 2 \mathrm{cc}$. mallein. \\
\hline$"$ & 3.o p.m. & $\mathrm{IOI}_{4}$ & \\
\hline$" \quad "$ & 7.0 p.m. & $102 \cdot 3$ & Very large swelling at seat of injection. \\
\hline$"$ & I I.O p.m. & $102 \cdot 3$ & \\
\hline " I6 & 3.0 a.m. & $102 \cdot 3$ & \\
\hline
\end{tabular}

Killed on the 23 rd January, and found to be glandered. 
CASE XXXIV.-Bay gelding; swollen legs and nasal discharge. Injected with mallein on the $15^{\text {th }}$ January.

\begin{tabular}{|c|c|}
\hline Hour. & Temperature. \\
\hline I r.o a.m. & 102.0 \\
$3.0 \mathrm{p.m}$. & $103 . \mathrm{I}$ \\
$7.0 \mathrm{p.m}$. & $104^{\circ} \mathrm{O}$ \\
I I.0 p.m. & $105^{\circ} \mathrm{O}$ \\
$3.0 \mathrm{a.m}$. & $105^{\circ} \mathrm{O}$ \\
\hline
\end{tabular}

Killed on the Igth January and found to be glandered.

CASE XXXV.-Bay gelding ; recently convalescent from purpura after strangles; submaxillary and prescapular glands swollen on off side. Injected with mallein Ioth December.

\begin{tabular}{|c|c|}
\hline Hour. & Temperature. \\
\hline 4.0 p.m. & 100.5 \\
8.0 p.m. & 103.5 \\
I 2.0 midnight & 1045 \\
9.0 a.m. & 104.0 \\
\hline
\end{tabular}

The horse was killed a few days later; the post-mortem revealed some pleuritic thickening and one small nodule of a doubtful nature on one lung. The enlarged lymphatic glands contained no traces of softening or degeneration.

CASE XXXVI.-Bay gelding; injected with mallein on Ioth December.

\begin{tabular}{|c|c|}
\hline Hour. & Temperature. \\
\hline $4.0 \mathrm{p.m}$. & $103^{\circ} 4$ \\
$8.0 \mathrm{p.m.}$ & $103^{\circ} 0$ \\
$12.0 \mathrm{midnight}$ & $105^{\circ} \mathrm{O}$ \\
$9.0 \mathrm{a} . \mathrm{m}$. & $105^{\circ} 5$ \\
\hline
\end{tabular}

Killed on the I6th December, and found to be badly glandered. 
CASE XXXVII.-Bay mare; suspected on account of general unthriftiness. Injected with mallein Ioth December.

\begin{tabular}{|c|c|}
\hline Hour. & Temperature. \\
\hline 4.0 p.m. & $100^{\circ} 0$ \\
$8.0 \mathrm{p.m}$. & $10 \mathrm{I}^{\circ} \mathrm{O}^{\circ}$ \\
I2.0 midnight & $103^{\circ} \mathrm{O}$ \\
$9.0 \mathrm{a.m.}$ & $105^{\circ} 2$ \\
\hline
\end{tabular}

Second injection on I 8 th February.

\begin{tabular}{|c|c|}
\hline Hour. & Temperature. \\
\hline Io.30 a.m. & 100.5 \\
4.30 p.m. & 100.5 \\
I0.0 p.m. & 103.2 \\
\hline
\end{tabular}

The mare was killed 12 hours after the second injection, and found to be glandered.

CASE XXXVIII.-Bay gelding; discharge from one nostril and enlarged submaxillary gland. Injected with mallein r 8 th February.

\begin{tabular}{|c|c|}
\hline Hour. & Temperature. \\
\hline I0.30 a.m. & $100 \%$ \\
4.30 p.m. & $104^{\circ} 0$ \\
I0.0 p.m. & $105^{\circ} \circ$ \\
5.0 a.m. & $103{ }^{\circ} 0$ \\
I I.1 15 a.m. & 104.6 \\
\hline
\end{tabular}

This animal has since been killed, and found to be glandered. 
CASE XXXIX.-Bay gelding; chronic thickening of fore leg, and losing condition. Injected with mallein I sth February.

\begin{tabular}{|c|c|}
\hline Hour. & Temperature. \\
\hline $10.30 \mathrm{a} . \mathrm{m}$ & 100.5 \\
\hline 4.3० p.m. & IOI 5 \\
\hline 10.0 p.m. & $104 \%$ \\
\hline 5.0 a.m. & $103^{\circ} \circ$ \\
\hline I I. I 5 a.m. & $101 \% 6$ \\
\hline
\end{tabular}

Killed about a week later and found to be very badly glandered.

CASE XL.-Grey gelding; suffering from disease of the foot; not suspected of glanders. Injected with mallein $23 \mathrm{rd}$ January.

\begin{tabular}{|c|c|}
\hline Hour. & Temperature. \\
\hline I0.0 a.m. & IOr. \\
4.0 p.m. & 104.0 \\
I I.30 p.m. & 105.5 \\
I0.0 a.m. & 105.5 \\
\hline
\end{tabular}

Killed a few days later and found to have numerous glanders nodules in lung.

CASE XLI.-Bay mare, in very low condition, and been isolated for nine weeks on account of nasal discharge and glandular enlargement. Injected with mallein 7 th February.

\begin{tabular}{|c|c|}
\hline Hour. & Temperature. \\
\hline I 1.0 a.m. & $\mathrm{I01}{ }^{\circ}$ \\
4.30 p.m. & 102.6 \\
I1.0 p.m. & 103.5 \\
4.0 a.m. & 104.0 \\
11.30 a.m. & 102.5 \\
\hline
\end{tabular}

Since killed, and found to be badly glandered. 
CASE XLII.-Bay gelding; unthrifty ; submaxillary and prescapular glands enlarged. Injected with mallein 7 th February.

\begin{tabular}{|c|c|}
\hline Hour. & Temperature. \\
\hline I I.0 a.m. & $99^{\circ} 6$ \\
5.0 p.m. & I01.0 \\
I I.0 p.m. & I05.0 \\
I I.30 a.m. & $105^{\circ} 5$ \\
\hline
\end{tabular}

Since killed and found to be badly glandered.

CASE XLIII.-Chestnut gelding; believed to be glandered. Injected with mallein $7_{\text {th }}$ February.

\begin{tabular}{|c|c|}
\hline Hour. & Temperature. \\
\hline I0.0 a.m. & $101^{\circ} 5$ \\
4.0 p.m. & 104.0 \\
I I.30 p.m. & 105.5 \\
10.0 a.m. & 104.6 \\
\hline
\end{tabular}

Since killed, and found to be glandered in lungs.

CASE XLIV.-Grey mare, in very poor condition; double nasal discharge. Injected with mallein I8th February.

\begin{tabular}{|c|c|}
\hline Hour. & Temperature. \\
\hline I I.1 5 a.m. & 102.0 \\
5.0 & p.m. \\
I I.0 p.m. & 103.5 \\
4.0 a.m. & 103.5 \\
I I.30 a.m. & 102.5 \\
\hline
\end{tabular}


The same mare received a second dose of mallein on the I 8 th February.

\begin{tabular}{|c|c|}
\hline Hour. & Temperature. \\
\hline $10.30 \mathrm{a.m}$. & 103.0 \\
\hline 4.3० p.m. & 103.5 \\
\hline 10.0 p.m. & IO4\% \\
\hline 5.0 a.m. & $102^{\circ} \mathrm{O}$ \\
\hline I I.O a.m. & $102{ }^{\circ} 0$ \\
\hline
\end{tabular}

Since killed and found to be badly glandered.

CASE XLV.-Bay gelding, the subject of incurable disease of the hip joint.

\begin{tabular}{|c|c|c|c|}
\hline Date. & Hour. & $T e m p$ & Remarks. \\
\hline Dec. 24 & 4.0 p.m. & $10 I^{\circ} 5$ & Received $21 / 2$ cc. mallein. \\
\hline$" \quad "$ & 9.0 p.m. & $10 I^{\circ} 0$ & \\
\hline$" 25$ & I.o a.m. & $103 \% 2$ & Yery clioht local swelling and no exi \\
\hline " & 6.0 a.m. & $103 \cdot 5$ & dence of general disturbance. \\
\hline$"$ & I I.O a.m. & $103^{\circ} \circ$ & \\
\hline
\end{tabular}

Killed about a week after injection. The post-mortem revealed no glanders lesions.

CASE XLVI._Black gelding, with open joint. Injected with mallein 2nd March, at IO.30 A.M.

\begin{tabular}{|c|c|}
\hline Hour. & Temperature. \\
\hline I0.30 a.m. & IOI'5 \\
5.0 p.m. & IOI'0 \\
I I.30 p.m. & IO3.0 \\
5.0 a.m. & IOI'0 \\
I I.0 a.m. & IOO'5 \\
\hline
\end{tabular}

The local reaction was slight, and there was no general disturbance. The animal was killed a few days later, and the post-mortem revealed no glanders lesions. 
CASE XLVII.-Bay mare, suspected on account of double nasal discharge. Injected with mallein on Ioth December, at 4 P.M.

\begin{tabular}{|c|c|}
\hline Hour. & Temperature. \\
\hline 4.0 p.m. & 10I.6 \\
8.0 p.m. & 101.5 \\
I2.0 midnight & I04.5 \\
9.0 a.m. & I06.0 \\
\hline
\end{tabular}

Killed a few days later. No glanders lesions were discoverable in the lungs or nasal septum. Unfortunately the submaxillary glands and the sinuses of the head were not examined.

CAsE XLVIII.-Grey gelding; subject of farcy; suspected of being glandered in lungs.

\begin{tabular}{|c|c|c|c|c|}
\hline \multicolumn{2}{|c|}{ Date. } & Hour. & Temp. & Remarks. \\
\hline Feb. & I 8 & 5.30 p.m. & $102 \%$ & Received $21 / 2$ cc. mallein. \\
\hline$"$ & $"$ & I I.3O p.m. & $103^{\circ} 4$ & \\
\hline$"$ & 19 & 3.30 a.m. & $105 \cdot 8$ & Iong soullinor \\
\hline$"$ & $"$ & 7.30 a.m. & IO 4.4 & $\begin{array}{l}\text { inches in diameter. Appetite main- } \\
\text { tained. }\end{array}$ \\
\hline$"$ & $"$ & I 1.50 a.m. & $\mathrm{IO}_{4}^{\circ} \mathrm{O}$ & \\
\hline$"$ & , & 3.3० p.m. & $103^{\circ} 2$ & \\
\hline
\end{tabular}

Killed a few days later and found to be glandered.

CASE XLIX.-Bay mare; thickening of near hind leg like lymphangitis; no cording of the lymphatics; submaxillary glands normal; farcy bud on off shoulder; slight discharge from near nostril ; short of breath on exertion.

\begin{tabular}{|c|c|c|c|}
\hline Date. & Hour. & $T_{c m p}$ & Remarks. \\
\hline Feb. 28 & I 1.0 a.m. & IOI'O & Received $2 \mathrm{~T} / 2 \mathrm{cc}$. mallein. \\
\hline " & 4.0 p.m. & 102.0 & $\ldots$ \\
\hline$" \quad$, & 8.o p.m. & 104.8 & \\
\hline$"$, & I 2.0 midnight & $105^{\circ} 0$ & $\begin{array}{l}\text { Large diffuse swelling at seat of inocula- } \\
\text { tion. }\end{array}$ \\
\hline$\Rightarrow 29$ & 4.0 a.m. & $103^{\circ} 0$ & \\
\hline " & I I.o a.m. & $103^{\circ} 0$ & $\ldots$ \\
\hline
\end{tabular}

Killed. Lungs full of glanders tubercles. 
CASE L.-Brown gelding; sticky fotid discharge from off nostril, and enlarged submaxillary gland; feeding well ; diagnosed ozæna.

\begin{tabular}{|c|c|c|c|c|}
\hline Date & & Hour. & Temp. & Remarks. \\
\hline Mar. & & 9.0 a.m. & $99^{\circ} 4$ & Received $21 / 2$ cc. mallein. \\
\hline$"$ & $"$ & 3.0 p.m. & $100 \cdot 6$ & $\cdots$ \\
\hline$"$ & $"$ & 7.0 p.m. & $101 \cdot 5$ & $\cdots$ \\
\hline$"$ & $"$ & 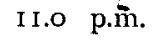 & IOI'O & $\cdots$ \\
\hline$"$ & 2 & 3.0 a.m. & $100 \% 4$ & $\ldots$ \\
\hline$"$ & $"$ & 7.0 a.m. & $100 \cdot 2$ & No swelling at seat of injection. \\
\hline$"$ & $"$ & I 1.30 a.m. & IOI ${ }^{\circ}$ & $\cdots$ \\
\hline$"$ & $"$ & 3.0 p.m. & IOI'O & $\ldots$ \\
\hline
\end{tabular}

A few days later the head was trephined and pus evacuated from the sinuses; case doing well.

Of the 50 horses referred to in the preceding pages, 42 have since been submitted to a post-mortem examination and 8 are still alive.

Dealing in the first place with the 42 that have died or been killed, an analysis of the foregoing records shows that 30 were undoubtedly glandered, 9 were apparently free from glanders lesions, and 3 (Cases X., XXXV., and XLVII), owing to incomplete examination or uncertainty regarding the nature of the lesions, remain doubtful. With one exception (Case XXXIII.) the temperature in the glandered horses rose to $103^{\circ}$ at least, and in all but 3 it rose to $104^{\circ}$ or more. It is worthy of note that in two of the three cases in which the reaction did not reach $104^{\circ}$ (Cases IV. and XLIV.) the temperature was already slightly febrile before injection, viz. $102^{\circ}$. In the third Case (XXXIII.) the initial temperature was IOI $2^{\circ}$, and after injection it rose to $102 \cdot 3^{\circ}$ only, but the local reaction was very marked. It may thus be said that the glandered horses without exception reacted to the mallein.

Taking next the 9 horses in which the post-mortem revealed no glanders lesions, it will be found that in only 4 of them did the temperature after injection reach $103^{\circ}$. The first of these was Case XII., in which after injection the temperature went up to $105^{\circ}$, but the local reaction was very slight. Moreover, after a second injection the maximum temperature recorded was only $1028^{\circ}$. The second was Case XXV., in which the temperature rose from $102^{\circ}$ to to $104^{\circ} 8^{\circ}$; in that case also there was very slight local reaction. In the third instance-Case XLV.- the initial temperature was $1015^{\circ}$, and after injection it rose to $103.5^{\circ}$, but the local reaction was very slight. Lastly, in Case XLVI. the temperature rose from IOI $5^{\circ}$ to IO $3^{\circ}$, but here again there was very little local reaction.

Coming lastly to the 9 animals that are still alive and at the present time judged to be free from glanders, it will be observed that in none 
of these did the temperature after injection rise to $102^{\circ}$. It must be admitted that to base a conclusion regarding the value of mallein on the assumption that these 9 animals are not glandered would be to employ a method of reasoning not altogether free from fallacy, but, on the other hand, quite apart from the fact that these horses exhibited no reaction after mallein, there is a very strong presumption that they are, and were at the time of injection, free from glanders.

The foregoing experiments, even taken by themselves, prove that in mallein there has been discovered an agent of incomparable value in the diagnosis of glanders. That it will prove infallible is not to be expected, but it may with confidence be pronounced the most reliable guide for even an experienced practitioner. It would be transparently foolish to trust blindly to the indications afforded by mallein, and to neglect altogether those means of arriving at a diagnosis that have hitherto had to be relied upon. It is not at all unlikely that mistakes will still be made in the diagnosis of glanders, but the practitioner who adds the use of mallein to his former methods of arriving at a decision in doubtful cases will make fewer mistakes than he did before. Every one knows that two different kinds of mistakes are made in such doubtful cases. The first, and the one that is generally considered the more serious mistake, consists in declaring a non-glandered horse to be glandered and advising his slaughter. This is perhaps not a common mistake, but veterinary surgeons who have to deal with large infected studs can hardly avoid committing it except by falling into the other error-no less serious as regards its consequences-of failing to recognise glanders until it has been months in existence. Both of these mistakes can be made very rare by systematically employing mallein on every horse that presents the clinical signs of glanders, and on every horse that is known to have been exposed to the contagion of glanders.

The very numerous observations that have now been published at home and abroad regarding the action of mallein appear to warrant the following conclusions :-

I. If in any horse that presents symptoms exciting a suspicion of glanders, or that is known to have been exposed to the infection of glanders, an injection of mallein raises the temperature from about the normal (under $\mathrm{IOI}^{\circ}$ ) to $\mathrm{IO}^{\circ}$, and produces a marked inflammatory reaction at the seat of injection, that horse may be pronounced "glandered."

2. If in such a horse an injection of mallein determines a marked local reaction and an elevation of temperature equal to $2^{\circ}$, that horse may be pronounccd " probably glandered."

3. In horses that have already a febrile temperature ( $102^{\circ}$ or over) the temperature reaction after mallein is inconstant, and unreliable as a guide to diagnosis.

It only remains to be added that the local reaction deserves to be called " marked" when the swelling extends for 3 or 4 inches from the point of injection.

In concluding this article I desire to express my best thanks to Messrs A. Rogerson, P. M'Onie, H. W. Caton, and other members of the profession in London, who have kindly afforded me the opportunity of observing the effects of mallein in cases under their care. I 
am also greatly indebted to Mr F. Hobday, late Hospital Surgeon ; Mr P. Falshaw, present Hospital Surgeon; Mr W. B. Edwards, clinical assistant; and $\mathrm{Mr} \mathrm{A}$. P. Calestremé, veterinary student, for the trouble they have taken in taking the temperature of the horses under observation.

\section{EDITORIAL ARTICLEx}

COW SCARLATINA AGAIN.

IT is probable that there are very few readers of this Journal who think it worth while to seriously discuss whether the disease termed scarlatina in Man attacks animals of the bovine species, or is ever in natural circumstances transmitted from the one species to the other. Dr Klein's remarkable series of investigations in connection with this subject may for a short time have inclined a good many people to believe that veterinary surgeons and stockowners had overlooked the existence of cow scarlatina until Dr Klein discovered it at Hendon, and that medical men had been looking in quite a wrong direction for the main cause of scarlatina among human beings; but at the present time we doubt whether there could be found anywhere a veterinary surgeon, or outside the British Islands a medical man, who believes that the examination of cows' teats forms a necessary part of every investigation regarding the source of infection in outbreaks of human scarlatina. This attitude of unbelief is not so much due to a conviction that Dr Klein's theory was disproved by the hostile criticism directed against it in the repeated discussions that centred round the Hendon inquiry, as to a feeling that if the theory had been well founded numerous instances of cow scarlatina, and of human epidemics traceable to them, would ere this have been brought to light either at home or abroad. For the last few years bovine scarlatina has been a muchsought-after disease, and yet we know that Dr Klein has had almost a monopoly in the discovery of cases. It cannot be denied that this is very difficult to reconcile with the notion that the Hendon disease was scarlatina, for whether we suppose that the bovine affection is in a manner natural to that species, like pleuro-pneumonia or footand-mouth disease, or that outbreaks of it are first started by infection from human beings suffering from scarlatina, the occurrence of the disease ought not to be rare.

During the past year there was discovered in the neighbourhood of Glasgow an outbreak of udder eruption that at first promised to lend support to Dr Klein's conclusions regarding the relationship between human scarlatina and a disease of cows. A report bearing on this outbreak and its supposed connection with a simultaneous epidemic of 